\title{
MORPHOLOGICAL FEATURES OF DENTOFACIAL AREA IN PEOPLE WITH DENTAL ARCH ISSUES COMBINED WITH OCCLUSION ANOMALIES
}

\section{S.B. Fischev', M.N. Puzdyryova', S.V. Dmitrienko², D.A. Domenyuk ${ }^{3}$, A.A. Kondratyuk ${ }^{4}$}

\author{
${ }^{1}$ Department of pediatric dentistry and orthodontics Saint-Petersburg \\ State Pediatric Medical University, Saint-Petersburg, Russia, st. \\ Lithuanian, 2, 194100.E-mail:spb@gpma.ru, tel: +7812 2950646. \\ ${ }^{2}$ Department of Dentistry, Pyatigorsk Medical-Pharmaceutical Institute \\ (Branch of Volgograd State Medical University, Ministry of Healthcare, \\ Russian Federation, 11, pr. Kalinina, Pyatigorsk-32, Stavropol Region, \\ Russia 357532.E-mail:s.v.dmitrienko@pmedpharm.ru, \\ tel: +78793324474 \\ ${ }^{3}$ Department of general practice dentistry and child dentistry, Stavropol \\ State Medical University, Ministry of Healthcare of Russian Federation, 310, \\ Mira Street, Stavropol, Russia 355017. E-mail: domenyukda@mail.ru, \\ tel: +79188701205 \\ ${ }^{4}$ Department of Dentistry Saint-Petersburg State Pediatric Medical \\ University, Saint-Petersburg, Russia, st. Lithuanian, 2, 194100. \\ E-mail:spb@gpma.ru, tel: +78122950646
}

The level of development of each era determines the specifics of recognizing diseases and teaching about them. Nowadays, there is an increased interest taken by patients in their own appearance as well as in the structural features of the facial part of their heads $[1,2]$. Another issue currently faced by the dentistry is the growing prevalence of dentofacial anomalies and deformities among various population groups $[3,4]$. The etiological factors behind anomalies and deformities include dental system congenital and acquired pathologies, and especially dental arch defects $[5,6,7]$. The effect of the dental arches pathology on the craniofacial status has been proven in numerous works written by clinical experts $[8,9]$. It has been noted that the timely treatment and preventive measures offered to patients with dental arches defects through different age periods, has a beneficial effect on the growth, development and condition not only of the masticatory system, yet also on the adjacent organs and body systems $[10,11,12]$. Given the above, studying maxillofacial morphology in people with dental arch defects will remain an urgent issue for dentistry.

Aim of study

To identify anatomical and physiological dentofacial features in people with dental arch defects combined with occlusion anomalies.
Article history:

Received 11 February 2019

Received in revised form 15 March 2019 Accepted 21 March 2019

\section{MATERIAL AND METHODS}

The maxillofacial area status was assessed in 312 people in their first mature period (21-35 years of age). Out of the total number of patients, a representative sampling was done involving 127 patients who needed pre-prosthetic orthodontic treatment. Subjective and objective research methods were employed following the protocol for the managing patients with partial absence of teeth.

\section{RESULTS AND DISCUSSION}

127 people out of 312 patients had a combined pathology, which accounted for $40.7 \pm 0.16 \%$. Out of the patients with dental arches defects in combination with occlusion pathology, 50 patients were found to have individual teeth position anomalies, which was $39.4 \pm 0.38 \%$ of the number of patients with combined pathology. Dental arches anomalies and deformities were observed in 35 people $(27.56 \pm$ $0.35 \%$ ), while another 42 patients had pathological occlusion, which in relative terms was $33.1 \pm 0.37 \%$ of the number of patients with the dental system combined pathology. Anatomical and physiological maxillofacial features, as a rule, had a pathology localization in the dental arch. Anomalies and deformities of the dental arches anterior part entailed aesthetic and functional impairments. There were frequent speech disorders and disturbed food bite. An incorrect position of the front teeth was the etiological factor behind periodontal disease accompanied by impaired blood circulation, while it contributed to the development of traumatic occlusion. There was pathological teeth mobility observed as well as disturbed teeth position in different directions. Patients with anomalies and deformities in the chewing segment, experienced discomfort in the mouth while chewing food. There was frequent overload of antimers, which suffered from additional stress in case of unilateral defects. In case of bilateral defects of the dental arches, patients often complained of the functional status of the temporomandibular joints. Patients with abnormal eruption terms, abnormalities in the number of teeth (hypodentia), and abnormal position of the teeth, 
were found to have varying degrees of teeth deviation from their normal position.

\section{CONCLUSION}

The results of the study indicate a high level of occlusion anomalies prevalence in people with dentofacial arches defects. Anatomical and physiological dentofacial features were typically attributed to the dental arch pathology localization.

\section{REFERENCES}

1. Domenyuk D., Porfyriadis M., Dmitrienko $S$. Major telerenthengogram indicators in people with various growth types of facial area. Archiv EuroMedica, 2018; Vol. 8; 1: 19-24.

2. Shkarin V., Domenyuk D., Lepilin A., Fomin I., Dmitrienko S. Odontometric indices fluctuation in people with physiological occlusion. Archiv EuroMedica, 2018; Vol. 8; 1: 12-18.

3. Borodina V.A., Domenyuk D.A., VeIsgeim L.D., DMITRIENKo S.V. Biometry of permanent occlusion dental arches - comparison algorithm for real and design indicators. Archiv EuroMedica, 2018; Vol. 8; 1 : $25-26$.

4. Korobkeev A.A., Domenyuk D.A., ShKarin V.V., DMitrienko S.V. Types of facial heart depth in physiological occlusion. Medical news of North Caucasus. 2018. - Vol. 13. - № 4. - P. 627-630. (In Russ., English abstract). DOI - https://doi.org/10.14300/ mnnc.2018.13122

5. Dmitrienko S.V., Domenyuk D.A. Dentoalveolar specifics in children with cleft palate during primary occlusion period. Archiv EuroMedica, 2018; Vol. 8; 1: 33-34.

6. DOMENYUK D.A., LePILIN A.V., Fomin I.V., DMITRIENKo S.V. Improving odontometric diagnostics at jaw stone model examination. Archiv EuroMedica, 2018; Vol. 8; 1: 34-35.

7. PorfiRiadis M.P., DMitrienko S.V., Domenyuk D.A., BudAichiev G.M.A. Mathematic simulation for upper dental arch in primary teeth occlusion. Archiv EuroMedica, 2018; Vol. 8; 1:36-37.

8. LePILIN A.V., Fomin I.V., DoMENYUK D.A., DMITRIENKo S.V. Diagnostic value of cephalometric parameters at graphic reproduction of tooth dental arches in primary teeth occlusion. Archiv EuroMedica, 2018; Vol. 8; 1: 37-38.

9. ShKarin V.V., Davydov B.N., Domenyuk D.A., DMITRIENKo S.V. Non-removable arch orthodontic appliances for treating children with congenital maxillofacial pathologies - efficiency evaluation. Archiv EuroMedica, 2018; Vol. 8; 1:97-98.

10. Korobkeev A.A., Domenyuk D.A., ShKarin V.V., DMitrienKo S.V., Weisheim L.D., KonNOV V.V. Anatomical features of the interdependence of the basic parameters of the dental arches of the upper and lower jaws of man. Medical news of North Cauca- sus. 2018. - Vol. 13. - № 1-1. - P. 66-69. (In Russ., English abstract). DOI - https://doi.org/10.14300/ mnnc.2018.13019

11. DMitrienko S.V., Davydov B.N., V.V. ShKarin, DOMENYUK D.A. Algorithm for determining the size of artificial teeth by the morphometric parameters of the face in people with full adentia. Dentistry. 2018; 97(6): 57-60

12. Domenyuk D.A., Zelensky V.A., DMitrienko S.V., Anfinogenova O.I., PushKin S.V. Peculiarities of phosphorine calcium exchange in the pathogenesis of dental caries in children with diabetes of the first type. Entomology and Applied Science Letters. 2018; 5(4): 49-64. 